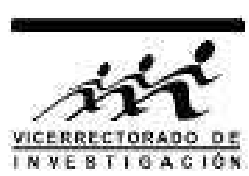

\title{
Experimentando el circuito de Chua
}

\author{
R. A. Montalvo y P. H. Rivera* \\ Facultad de Ciencias Físicas, Universidad Nacional Mayor de San Marcos, Lima, Perú
}

Recibido 20 noviembre 2016 - Aceptado 15 diciembre 2016

\begin{abstract}
En los últimos años de crisis económica, estamos considerando diseñar y construir equipamiento científico para enseñanza e investigación, usando los componentes electrónicos de código abierto que se han desarrollado en los últimos años precisamente por la crisis, como el Arduino y el Raspberry Pi, que se encuentran en el mercado a muy bajo costo.

En el presente trabajo, reportamos el comienzo de esta aventura, con la implementación de un circuito relativamente simple como el circuito de Chua, ampliamente conocido por presentar un comportamiento caótico por la no-linealidad formada por dos comportamientos lineales en un intervalo de voltaje.

Palabras claves: Circuito de Chua, sistema dinámico, caos clásico.
\end{abstract}

\section{Experimenting the Chua circuit}

In the last years of economical crisis, we are considering to design and build scientific equipment for learning and research purpose, using electronic devices with open code which have developed in the last years precisely by the crisis. such as the Arduino and the Raspberry Pi, which offers at low cost in the local market.

In the present work, we report the beginning of this adventure, with the implementation of a relatively simple circuit such as the Chua's circuit, broadly know to present a chaotic behaviour by the non-linearity formed by two linear behaviour in an interval voltages.

Keywords: Chua circuit, dynamical system, classic chaos.

La crisis económica mundial iniciada en el 2008, como siempre ha ocurrido en un ambiente de crisis, ha afectado a los fondos financieros que soportan el desarrollo científico en los diferentes países de todo el planeta. Especialmente, los fondos que se invierten en ciencia básica como la física.

En el Perú, a pesar del crecimiento moderado promedio que ha tenido la economía desde el 2012 hasta el presente, y a la decisión política de invertir en desarrollo científico, esto no ha implicado una inversión sostenida en ciencia básica que incluye a la física.

En el 2005, después del desarrollo de una tesis de maestría desarrollado por Hernando Barragán, bajo la asesoría de Massimo Banzi, quien organizó un grupo de estudiantes del Instituto IVREA, en Ivrea, Italia, para desarrollar un sistema de hardware y software de código abierto para instrumentación de laboratorio electrónico, se estableció el Arduino en homenaje al lugar del Bar di Re Arduino donde solían reunirse [1,2], después de laboriosos y arduos días de trabajo.

En este contexto de crisis, ha surgido una gran cantidad de proyectos usando el Arduino, así como otras plataformas de hardware como el Raspberry $\mathrm{Pi}$ 3, 4, con procesadores ARM que usan el Linux ARM con el objetivo de estimular la enseñanza en ciencias de la computación, pero que está siendo usado en proyectos más sofisticados como la construcción de un espectrómetro Raman de bajo costo [5].

El proyecto que estamos desarrollando es compilar toda la información que se encuentra en la literatura e Internet para aplicar en el diseño de algunos experimentos de física básica y la construcción de equipamiento de laboratorio usando el Arduino y otras plataformas semejantes al Raspberry $\mathrm{Pi}$, de muy bajo costo, que se encuentran en el mercado.

En este artículo, presentamos el uso del Arduino para el análisis de un sistema dinámico basado en el circuito de Chua [6, 7] que es el paradigma desde 1983 del comportamiento caótico de un circuito eléctrico. En primer lugar, hacemos una breve descripción del circuito, luego explicamos el proceso de simulación usando el Multisim y finalmente mostramos el circuito construído y las observaciones realizadas en este interesante experimento.

*priverar@unmsm.edu.pe 


\section{Diseño}

El sistema dinámico a analizar es un circuito eléctrico diseñado por Leon O. Chua en 1983, cuando realizaba una estadía de profesor visitante en la Waseda University, en Japón.

Hacemos un breve análisis del circuito de Chua basado en la descripción muy práctica publicada en la página web del chuacircuits.com [8]. Los lectores interesados pueden encontrar muy buena información en este sitio, para construir un circuito de Chua como experiencia demostrativa en sus clases de sistemas dinámicos. El circuito de Chua que se muestra en la figura 1 , consiste de un inductor $L$, dos capacitores $C_{1}$ y $C_{2}$, un diodo Chua $g\left(V_{1}\right)$ y una resistencia variable $R_{v}$, presenta las siguientes ecuaciones cuando usamos las leyes de los circuitos de Kirchhoff para el análisis del circuito,

$$
\begin{aligned}
C_{1} \frac{d V_{1}}{d t} & =\frac{1}{R_{v}}\left(V_{2}-V_{1}\right)-g\left(V_{1}\right) \\
C_{2} \frac{d V_{2}}{d t} & =\frac{1}{R}\left(V_{1}-V_{2}\right)+I_{L} \\
L \frac{d I_{L}}{d t} & =-V_{2} .
\end{aligned}
$$

donde la corriente $g\left(V_{1}\right)$ que atravieza el diodo de Chua está definido por

$$
g\left(V_{1}\right)=\left\{\begin{array}{cc}
G_{b} V_{1}+\left(G_{b}-G_{a}\right) E, & V_{1} \leq-E \\
G_{a} V_{1} & \left|V_{1}\right|<E \\
G_{b} V_{1}+\left(G_{a}-G_{b}\right) E & V_{1} \geq E
\end{array}\right.
$$

además $V_{1}, V_{2}$ son las diferencias de potencial en los capacitores $C_{1}$ y $C_{2}$, respectivamente. Mientras que $I_{L}$ es la corriente en el inductor $L . G_{a}$ y $G_{b}$ son las conductancias que estrictamente significan las pendientes de las rectas cuando se grafica la corriente $g$ versus $V_{1}$, el voltaje del capacitor $C_{1}$. Esta gráfica tiene dos pendientes diferentes y el punto de inflexión de las dos rectas ocurre en el voltaje $V_{1}=E$ y $V_{1}=-E$.

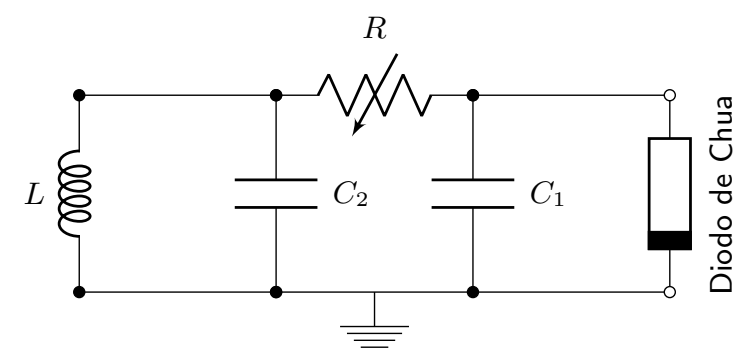

Figura 1: La forma más simple del circuito de Chua [8], incluyendo el diodo de Chua.

En este circuito bastante simple y sencillo de construir, la parte que incorpora el cambio de linealidad al sistema es el diodo diseñado por Chua que consiste de dos
OMPs1 encapsulados en un circuito integrado, $\mathrm{Cl}$, denominado TL082CP y seis resistencia $R_{x}$ de diferentes valores distribuídas entre los contactos del TL082CP tal como se muestra en la figura 2 .

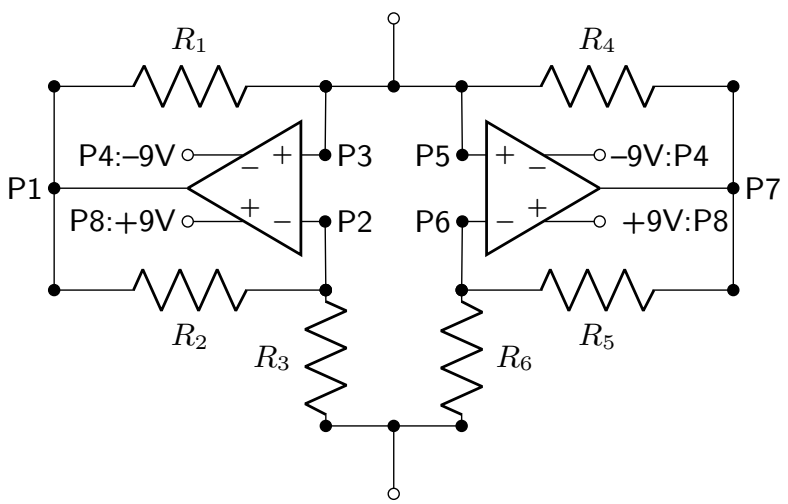

Figura 2: Circuito que genera el diodo de Chua [8]. Nótase que se usan 2 OMPs contenidas en el TL082CP y seis resistencias. Los terminales P1, P2, . , P7 y P8 corresponden a los contactos del CI TL082CP.

\section{Simulación}

Existe muchos programas que simulan los circuitos eléctricos. El precursor de ellos fue el Spice [9] creado en los ambientes del Department of Electrical Engineering and Computer Sciences de la University of California en Berkeley, este es un código abierto muy utilizado en la industria y en la academia.

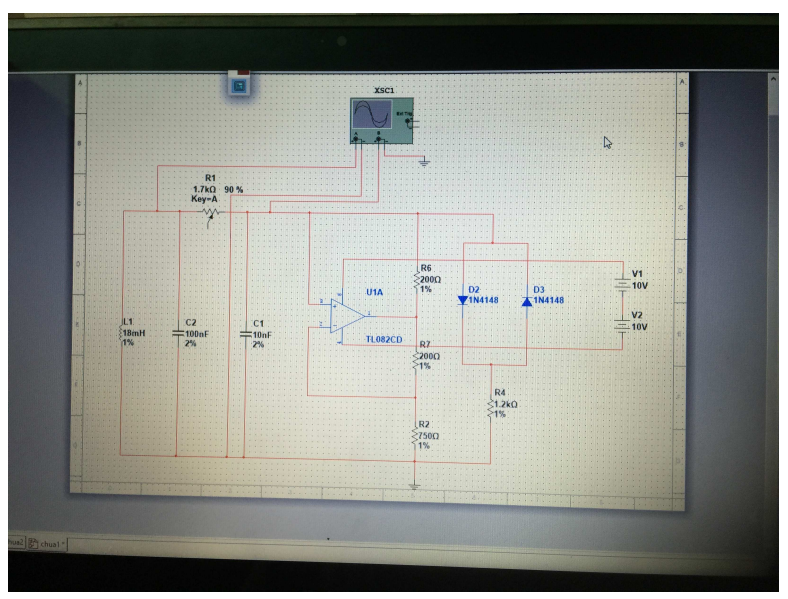

Figura 3: Diagrama del circuito Chua construído en el MultiSim de la National Instruments para simular su funcionamiento.

A partir del Spice se han desarrollado otros simuladores, que se caracterizan por ser más amistosos ante un usuario iniciante, algunos de ellos se encuentran en línea como el

\footnotetext{
${ }^{1}$ Amplificador operacional, Operational Amplifier.
} 
PartSim 10]. En el presente hemos usado el simulador Multisim 11 que es bastante amistoso para los usuarios que usan frecuentemente el Windows. En Linux está el propio Spice 3 con la interface de usuario Nutmeg, el Xcircuit que usa las librerias X11 y uno de los más recientes es el Quite Universal Circuit Simulator, Qucs [12].

El MultiSim utilizado en este trabajo es un simulador de la National Instruments, que nos permite construir el diseño del circuito en su panel principal con la libreria de componentes electrónicos de las diferentes empresas proveedoras, una muestra de nuestro simple circuito de Chua se muestra en la Figura 3.

Obsérvese que en el osciloscopio virtual, el canal A mide el voltaje $V_{A}$ entre el punto medio que conecta al inductor $L$, el capacitor $C_{1}$ y la resistencia variable $R_{v}$ y el punto a tierra, mientras que el canal $\mathrm{B}$ mide el voltaje $V_{B}$ entre el punto que une la resistencia conectada a una de las salidas del OMP TL082 y el capacitor $C_{2}$ y el punto a tierra. E CI TL082 posee dos OMP y la entrada de alimentación a los mismos usa un solo contacto positivo, $\mathrm{P} 8$, con $+9.0 \mathrm{~V}$ y un solo contacto negativo, $\mathrm{P} 4$, con $-9.0 \mathrm{~V}$, ambos son conectados a una fuente de $18 \mathrm{~V}$. Cabe señalar que las salidas de los dos OMPs son voltajes variables que alimentan al circuito de Chua, Figura 1. Otro punto de interés que se mide es la corriente que circula en el inductor $I_{L}$.

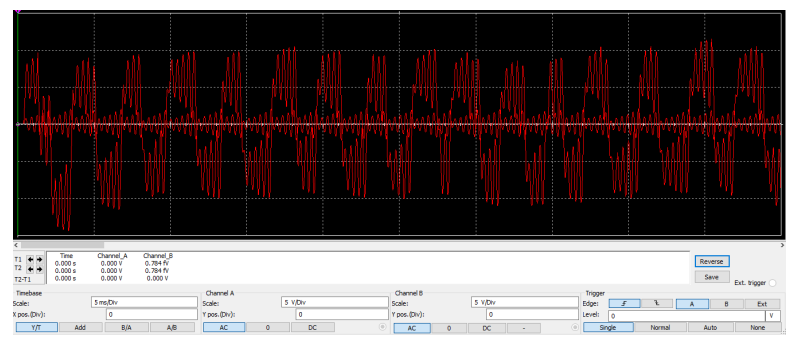

Figura 4: Se muestran las dos señales que se detectan en los canales $A$ y $B$ en el osciloscopio virtual usando el MultiSim, mostrando un comportamiento caótico.

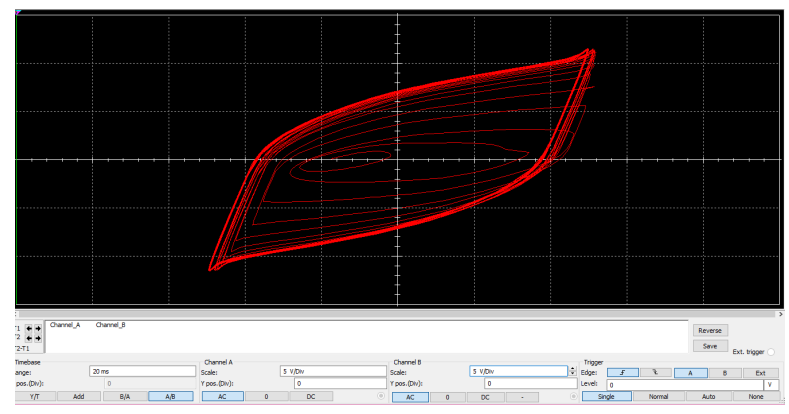

Figura 5: La superposición de los canales $A$ y $B$ en el plano $X Y$ en el osciloscopio virtual usando el MultiSim para diferentes corrientes permite la observación de un atractor.

En el proceso de la simulación se pueden cambiar los valores de todos los componentes, pero una vez fijados, la componente que varía es la resistencia variable $R_{v}$. La simulación y la adquisión de datos para los voltajes en los puntos $A$ y $B$ se muestra en la Figura 4.

La superposición de las señales adquiridos en los canales $A$ y $B$ se muestran en un plano $X Y$ donde los ejes $X$ e $Y$ corresponden a los canales $A$ y $B$, respectivamente. La observación del plano es desde una perspectiva ubicada en $Z$ que corresponde a la observación de la corriente que circula en el inductor $I_{L}$. Esto significa que las Figuras $5 \mathrm{y}$ 6 corresponde al matching de las señales $A$ y $B$ medidos en voltios y la formación de un o dos atractores corresponde a una variación cuidadosa de la resistencia variable $R_{v}$ que controla la circulación de corriente a los condensadores $C_{1}$ y $C_{2}$, que en el primer caso controla la corriente de la OMP en el diodo Chua y en el segundo, la corriente en el inductor $L$.

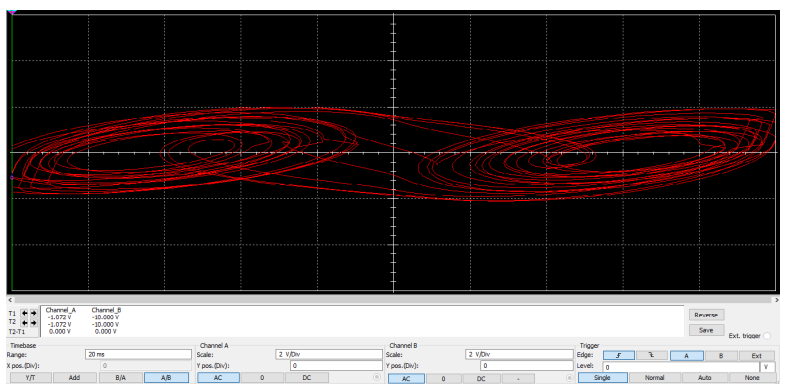

Figura 6: Modificando la resistencia $R_{v}$ permite que las dos señales que se detectan en los canales $A$ y $B$ en el plano $X Y$ en el osciloscopio virtual usando el MultiSim para diferentes corrientes permite la observación de dos atractores.

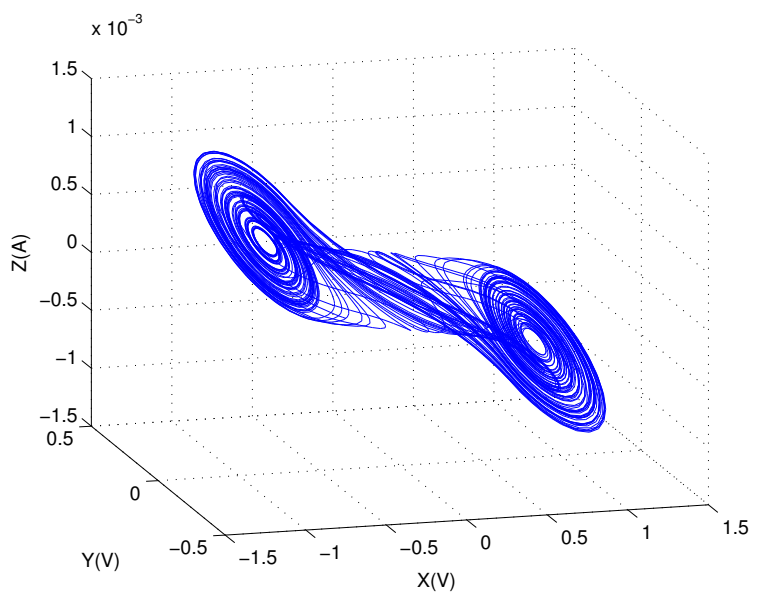

Figura 7: Esta es una simulación en el Matlab que permite observar la formación de los atractores en 3D, en el eje $X$ e $Y$ se encuentran los voltajes detectados a través de los canales $A$ y $B$, respectivamente. $\mathrm{Y}$ en el eje $\mathrm{Z}$, se observa la corriente $I_{L}$ en $\mathrm{mA}$.

En la Figura 7, presentamos una simulación 3D en Matlab producido con un código encontrado en el circuits- 
chua.com que muestra en el plano $X Y$ los voltajes de los canales $A$ y $B$ medida en voltios, mientras que, en el eje $Z$ se muestra la corriente en el inductor $I_{L}$ medida en $\mathrm{mA}$.

\section{Experimento}

En la implementación experimental de este circuito, hemos utilizado los componentes electrónicos que se encuentran en el mercado, la resistencia variable es de $5 \mathrm{k} \Omega$, las resistencias $R_{1}$ y $R_{2}$ son de $200 \Omega, R_{4}$ y $R_{5}$ son de 27 $\mathrm{k} \Omega$ y la resitencia $R_{3}$ de $220 \mathrm{k} \Omega$. El inductor es de $15 \mathrm{mH}$, los capacitores son $C_{1}=10 \mathrm{nF}$ y $C_{2}=100 \mathrm{nF}$ y el OMP utilizado es el TL082CD y dos baterias de $12 \mathrm{~V}$. Hemos utilizado una placa Arduino UNO y una placa Arduino ME$\mathrm{GA}$, ambos funcionan sin ningún problema en Linux. Como fuente de voltaje para alimentar las entradas de los OMPs se han usado hasta 2 baterias de $9 \mathrm{~V}$ en serie. Puesto que hay una alta atenuación en los OMPs, cuando la entrada es de $18 \mathrm{~V}$, la salidas están en torno de $2 \mathrm{~V}$ para el canal $A$ y en $\mathrm{mV}$ para el canal $B$.

Hemos utilizado un código en Python para adquirir los datos via el Arduino y hemos verificado su funcionamiento con un circuito RC y RLC cuyos gráficos no lo incluímos aquí por razones de espacio. El arreglo que incluye al breadborad donde hemos colocado los componentes para el circuito Chua, la placa Arduino y la computadora donde se encuentra el controlador del Arduino y el Python se muestran en la Figura 8.

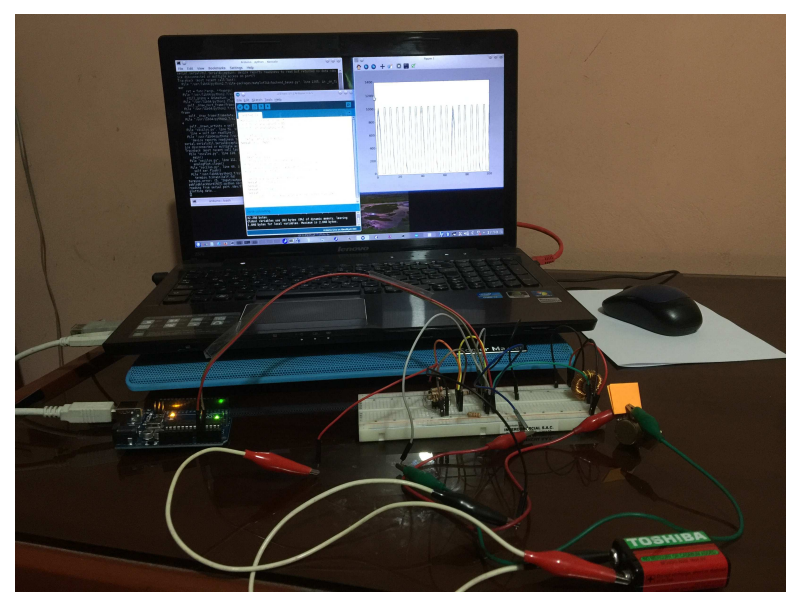

Figura 8: Se muestra el arreglo experimental para la adquisición de datos para el circuito Chua mostrado en el breadboard, en lado izquierdo la placa Arduino, y en el fondo la pantalla mostrando la ventana del controlador del Arduino y el programa en Python que grafica los datos.

En este experimento, por la simplicidad del circuito, puede parecer que es muy fácil de obtener los resultados, pero nos hemos encontrado con una dificultad en cuanto a que los componentes que se encuentran en el mercado tiene un error de $5 \%$ y la estabilidad mecánica de la resistencia variable no es muy confiable, puesto que no hemos conseguido observar dos atractores sino solamente uno, tal como se muestra en la Figura 9. Para este caso hemos utilizado un osciloscopio analógico del Laboratorio de Física III, HM303-6 de la Hameg Instruments.

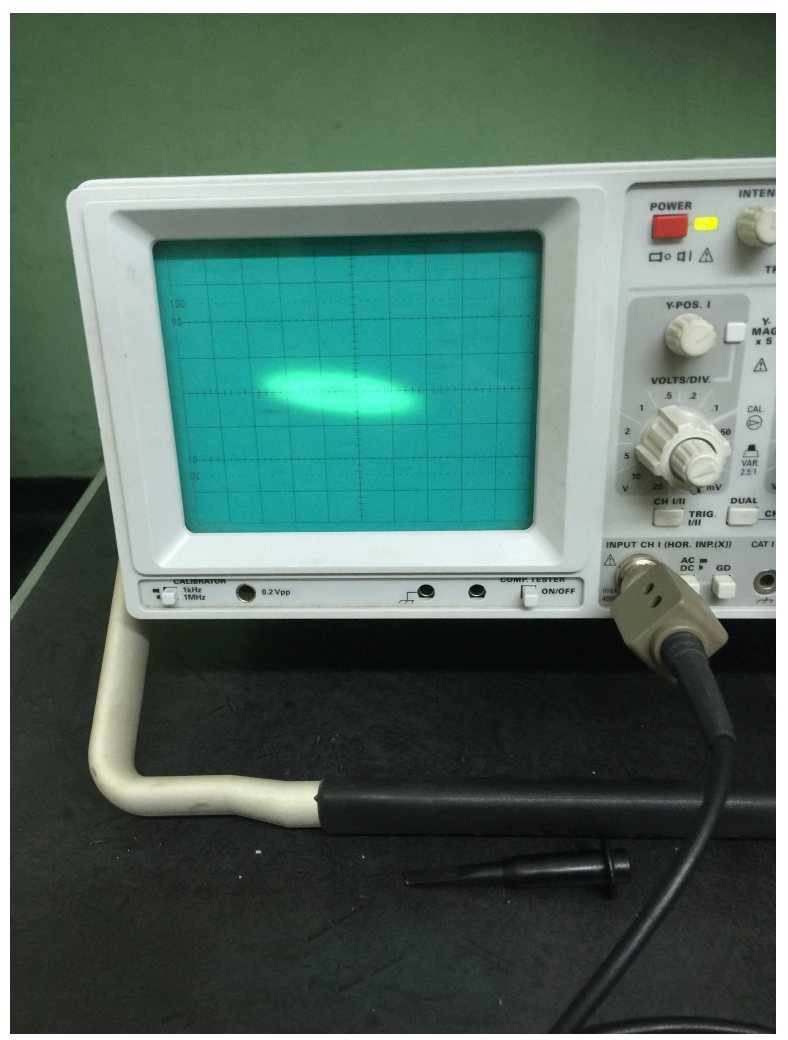

Figura 9: En el osciloscopio analógico HM303-6 de la Hameg Instruments observamos la formación de un atractor cuando hacemos el matching de las señales de los canales $A$ y $B$.

\section{Conclusiones}

Hemos implementado un circuito Chua utilizando todas las herramientas disponibles en Internet y hemos, al menos, observado cualitativamente a través de un osciloscopio analógico un atractor producido por el comportamiento caótico de este circuito. La resistencia variable del circuito juega un papel crucial en el experimento, la buena estabilidad mecánica y el rango de valores entre 0 y $2 \mathrm{k} \Omega$ asegura la observación del comportamiento caótico del sistema.

Esta experiencia nos abre las puertas para diseñar y construir dispositivos de medida de mayor envergadura tanto para la enseñanza de física básica como de investigación. 


\section{Referencias}

[1] es.wikipedia.org/wiki/Arduino

[2] www.arduino.cc/en/Guide/Introduction

[3] es.wikipedia.org/wiki/Raspberry_Pi

[4] www.raspberrypi.org/

[5] hackaday.io/project/1279-ramanpi-raman -spectrometer

[6] es.wikipedia.org/wiki/Circuito_de_Chua
[7] L. O. Chua, M. Komuro y T. Matsumoto, IEEE Trans. Circuits Syst. 33, 1072 (1986).

[8] www.chuacircuits.com

[9] bwrcs.eecs.berkeley.edu/Classes/IcBook/SPICE/

[10] https://www.partsim.com/simulator\#

[11] wWw.ni.com/multisim

[12] qucs.sourceforge.net/ 